\title{
Computational Model for Mechanics of Total Knee Replacement: Effect of Tibial Rotation during Deep Flexion in Relation to Post-Cam Design
}

\author{
Mohd Afzan Mohd Anuar1,4*, Mitsugu Todo², Ryuji Nagamine ${ }^{3}$ \\ ${ }^{1}$ Interdisciplinary Graduate School of Engineering Sciences, Kyushu Univerisity, 6-1 Kasuga-koen, Kasuga, \\ Fukuoka, 816-8580, Japan. \\ 2 Research Institute for Applied Mechanics, Kyushu University, 6-1 Kasuga-koen, Kasuga, Fukuoka, \\ 816-8580, Japan. \\ ${ }^{3}$ Sugioka Memorial Hospital, 3-6-1 Kashiiteruha, Higashi-ku, Fukuoka, 813-0017, Japan. \\ 4 Faculty of Mechanical Engineering, 40450 Universiti Teknologi MARA, Shah Alam, Selangor, Malaysia. \\ * Corresponding author. Tel.: +8192-583-7762; email: ar-m-afzan@mms.kyushu-u.ac.jp \\ Manuscript submitted July 28, 2014; accepted September 8, 2014. \\ doi: 10.17706/ijbbb.2014.4.6.428-437
}

\begin{abstract}
The requirement of deep kneeling is common among Asians e.g. Seiza and kneeling during prayer. In this study, the sensitivity of stress in toral knee replacement (TKR) post to tibial rotation during deep flexion was analysed and its relation to design of post-cam articulation was investigated. Three dimensional (3D) finite element models of two designs of clinically used posterior stabilized (PS) type total knee arthroplasty were constructed using their computational aided drawing (CAD) data. Loaded deep flexional motion from 0 to 135 degree in neutral, $10^{\circ}$ and $15^{\circ}$ of tibial rotation was adapted using finite element model to characterize the effects of knee kinematics on the stress states of ultra-high molecular weight polyethylene (UHMWPE) tibial inserts and relation to post-cam design. Peak equivalent stresses in post at neutral, $10^{\circ}$ and $15^{\circ}$ tibial rotation were $65.22 \mathrm{MPa}, 108.97 \mathrm{MPa}$ and 134.50 MPa respectively, for Superflex, and 47.16 $\mathrm{MPa}$, 45.69 $\mathrm{MPa}$ and 47.67 $\mathrm{MPa}$ respectively, for NRG. The result shows that the variation of maximum equivalent stress at different flexion angle and tibial rotation were caused by the post-cam contact geometry in sagittal plane and axial plane, respectively. Stress state of Superflex tibial post was found to be more sensitive to tibial rotation as compared to NRG. Modification on the post design based on post-cam radius of curvature ratio in both axial and sagittal has eliminated the sharp edge and provided larger contact area, hence reduce edge loading and high stress concentration area in Scorpio NRG. Stress in Scorpio NRG tibial post was found to be less sensitive to tibial rotation.
\end{abstract}

Key words: Deep flexion, finite element analysis, post-cam design, tibial rotation.

\section{Introduction}

In the recent years, due to the change of diet habits of elderly population, number of patients with osteoarthritis has been increasing every year. In most cases, total knee replacement (TKR) operation has been chosen as the last and most promising solution. In the United States, more than 400,000 TKR procedures have been carried out in 2003 and as reported in previous paper the number is predicted to increase exponentially within the next 30 years [1]. Quality of life of the patients who are suffered with severe osteoarthritis is found to be improved after being applied with total knee arthroplasty (TKA) 
procedure [2]. Posterior-stabilized (PS) type TKA was designed in such way to avoid posterior subluxation of tibia by means of post-cam mechanism to replace the function of posterior cruciate ligament while allowing natural femoral rollback [3]. In TKA implant design, durability and kinematics are two essential factors that must be taken into account. Design of TKA implant including the interfaces articulation, contact geometry and relative alignment between components with combination of surrounding soft tissues will determine the kinematics of the implant that leads to the durability of the UHMWPE tibial insert in terms of the stress states within the component [4], [5]. Via design alterations, function of TKA has been improved however for Asian daily activities, deep flexion has become main concern. For example, Japanese requires 150 degree of flexion for kneeling on tatami mat [2].

It is essential to well understand the stress state in polyethylene tibial insert during such kinematics as the tibial insert wear and failure due to post-cam contact under deep flexion has become crucial problem in PS type TKA. Three dimensional finite element analysis has been found to be a powerful tool in assessing the three dimensional stress distribution of implant knee system. In numerous investigations, normal walking gait was utilized as the input motion to quantify the kinematics and durability of TKR implant [4], [6]-[13]. Work done by Halloran et al. to develop and verify experimentally an explicit finite element (FE) TKR model that include articulations of tibio-femoral and patello-femoral [8]. The results have shown a good kinematic conformity between simulation and experimental measurement using Stanmore knee simulator in terms of anterior-posterior displacements and internal external rotations. In durability analysis, they have exhibited the effect of mesh size and rigidity of FE models on contact pressure and contact area. Meanwhile, Godest et al. have used explicit dynamic FE analyses to develop dynamic models of tibial insert-femoral articulations that able to determine the knee kinematics and contact mechanics simultaneously during dynamic-loading-controlled walking gait cycle conditions [4]. In other study, explicit finite element simulation has been utilized to study the effect of different medial:lateral loading ratio on the kinematics and stresses produced by TKR during normal gait cycle [13]. It was found that the von Mises stress and contact stress significantly affected by the small offsets of the vertical loading. The larger maximum von Mises stress was exhibited by the unicondylar loading (95:5 of medial:lateral loading ratio) as compared to bicondylar loading (50:50) with 4-6 MPa difference along the gait. The results however, have shown that the kinematics of TKR is less sensitive to the small changes of eccentric loading.

Kinetics analysis (force and pressure) of artificial knee joint undergo deep flexion motion was conducted to determine the effect of such kinematics to the performance of TKR in quite number of studies [2], [14]-[16]. Innocento et al. applied a numerical sensitivity analysis to investigate the effect anatomical factors: medial and lateral collateral ligaments location, patella tendon length; and component alignment: proximal-distal patellar orientation, patellar tilting and tibial component arrangement, for various types of knee implant on the patello-femoral and tibio-femoral articulations contact forces [15]. The knee joint was subjected with 10 seconds loaded $(200 \mathrm{~N})$ deep squatting between $0^{\circ}$ to $120^{\circ}$. Tibio-femoral contact force was found to be sensitive to the anterio-posterior displacement of medial collateral position with respect to femur and tibia. In different research, stress analysis of TKA undergoes three high flexion activities: stairs climbing, standing from a chair and standing from a double-leg kneeing was conducted [16]. Three different flexion angles: $60^{\circ}, 90^{\circ}$ and $135^{\circ}$; with femoral loads of $20 \%$ to $450 \%$ body weight acting inferiorly and posteriorly to represent those three different conditions of human activity. Huang et al. applied 500-N shear stress at $60^{\circ}, 90^{\circ}, 120^{\circ}$ and $150^{\circ}$ flexion to study the effect of post-cam design on the stress state of PS tibial post [14]. However, these studies only applied simple loading condition on the knee joint without taking into account in vivo data of deep flexional motion.

The effect tibial rotation on the stress state of TKR is less pronounced by the researchers. Previous study shows that tibial rotation at the highest flexion varies among different patients. Ueo et al. carried out an 
evaluation on the patients internal rotation who able to knee (seiza) after undergoing TKR operations [17]. The evaluation was performed to 30 knees from 23 patients and the outcome showed that the internal tibial rotation was ranging from $0.8^{\circ}$ to $26.7^{\circ}$ with average $14.3^{\circ}$ at full flexion. Research by Kanekasu et al. reported that the largest angle of tibial rotation acquired from 18 post-TKR patients was $17^{\circ}$ at $137^{\circ}$ flexion [18]. Kinematics characterization has been conducted also on intact knee joint. Nakagawa et al. performed a kinematics study on 20 Japanese subjects using MRI and found that there was a $13^{\circ}$ of tibial rotation when the knee moved from $133^{\circ}$ to $162^{\circ}$ flexion [19]. There are a few attempts have been done to study the kinetics of TKR (force \& pressure) undergo deep flexion motion combine with tibial rotation [3], [14]. However, these studies only investigate deep flexion with neutral and $10^{\circ}$ tibial rotation and most of them do not discuss further the influence of tibial rotation on the stress distribution of tibial insert and relation to post-cam design. In this study, two FE models of clinically used TKR, Stryker's Scorpio Superflex and Scorpio Non-Restrictive Geometry (NRG), respectively having different post-cam design were constructed. The models were subjected with dynamic loaded knee flexion between 0 to $135^{\circ}$ and tibial rotation of $0^{\circ}, 10^{\circ}$ and $15^{\circ}$ to analyze the sensitivity of stress in TKR post to tibial rotation and deep flexion.

\section{Method and Analysis}

There are two types of PS type knee prosthesis with different post-cam design have been analyzed. FE models were developed from the 3-D CAD data of both designs of prosthesis. The stiffness of femoral and tibial components is much higher in comparison to tibial insert, thus they have been treated as rigid bodies. The tibial insert was made of ultra-high molecular weight polyethylene (UHMWPE) and assumed to be elastic-plastic material.

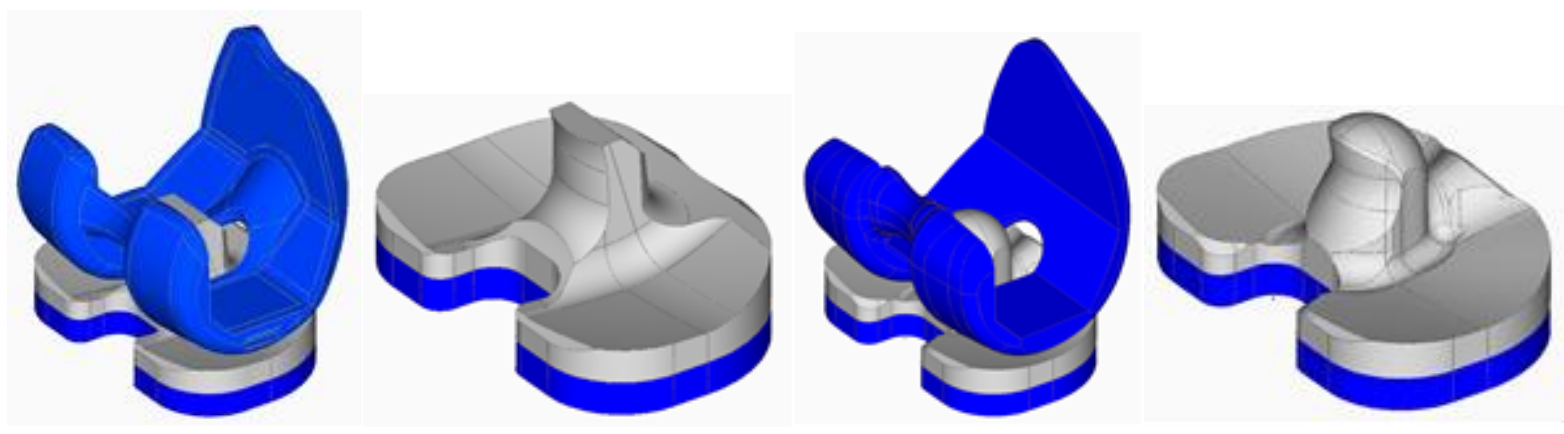

(a)

(b)

Fig. 1. CAD models of knee prosthesis (a) SuperFlex; and (b) NRG.

In Superflex and NRG, 143153 and 121538 split 4-node tetrahedron finite elements, respectively were utilized to represent the femoral component, tibial component and tibial insert. Fig. 1 shows the CAD model of both TKRs. The FE models were validated by comparing the peak contact stress, mean contact stress and contact area with the results obtained in previous study whereby a $500-\mathrm{N}$ compressive posterior load was applied to the tibial component against the femoral part [3]. In natural healthy knee joint, the effect of friction is almost negligible in dissimilarity to TKA motions that can be unsmooth [6]. Therefore, the coefficient of friction of 0.04 between femoral component and tibial insert was selected to be consistent with previous research [4], [8]. In actual TKA knee, flexing motion of femoral and tibial components are strongly influenced by the ligaments and muscles surrounding the knee, at the same instant balancing the reaction and frictional forces generated on the condylar and post surfaces of tibial insert. In the present models, two pairs of nonlinear springs were positioned anteriorly and posteriorly to the tibial insert to represent the action of those ligaments [12]. The nonlinear force-displacement relation of springs was given 
by:

$$
F=k_{1} d^{2}+k_{2} d=0.18667 d^{2}+1.3313 d
$$

Where $F$ is force exerted by the spring, $d$ is the displacement of the spring and $k_{1}$ and $k_{2}$ are the stiffness coefficients of the springs, respectively. To evaluate the model, input loadings and kinematics of normal walking was applied to determine the contact area and contact stress curve during one complete cycle.

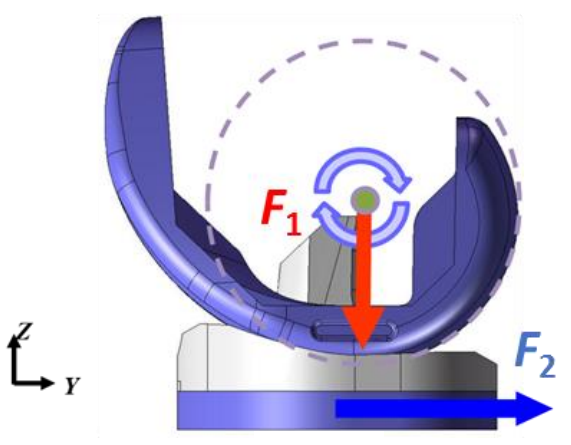

(a)

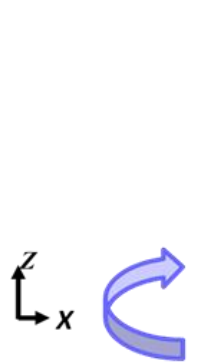

Fig. 2. Boundary conditions of TKA model (a) Sagittal view;

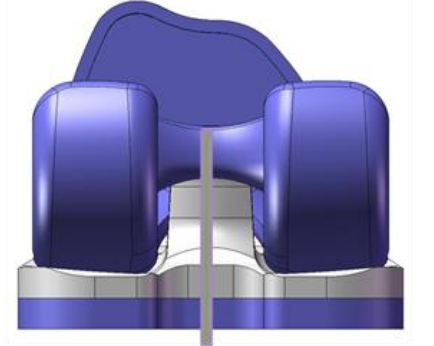

(b)

and (b) Posterior view.

In the present study, the TKRs were modeled to perform high flexion motion from 0 to $135^{\circ}$ with neutral, $10^{\circ}$ and $15^{\circ}$ tibial rotation. The displacement of femoral component was free in proximodistal axis, and fixed in mediolateral and anteroposterior axis. It was allowed to rotate about its mediolateral axis which was assumed to be coincide with the centre of curvature of the condylar surface of the femoral component. The tibial component was allowed to translate freely in anteroposterior direction and fixed in mediolateral and proximodistal axis. Fig. 2 shows the boundary conditions of the TKR model. At the same instant, load data for rapid deep squatting was applied [20]. The loadings, $F_{1}$ and $F_{2}$ represent the normal load and shear load sustained by the TKR. The force-flexion angle relation is shown in Fig. 3.

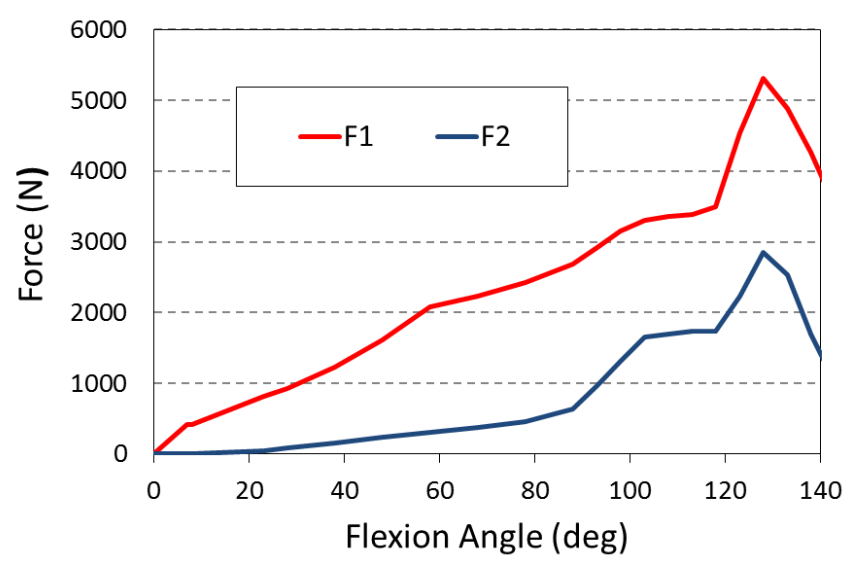

Fig. 3. Force-flexion angle relation of deep squatting.

\section{Results}

\subsection{Validation Test}

The validation test was carried out for flexion angles of $90^{\circ} \& 120^{\circ}$. Table 1 shows the comparison of peak contact stress, mean contact stress and contact area between present finite element model and result by Nakayama et al. [3]. The largest difference of peak contact stress, mean contact stress and contact area between FEM and experimental result by Nakayama et al. were 14.5\%, 17.1\%, 7.9\%, respectively showed a 
good agreement between both results. Due to different size and geometry of TKR design, direct comparison with previous explicit FE models [4], [8], [13] was intricate, however the evaluation test of present explicit FE models exhibited typical trends of maximum contact stress and contact area of normal walking gait as shown in Fig. 4.

Table 1. The comparison of peak contact stress, mean contact area and contact area

\begin{tabular}{|c|c|c|c|c|c|c|}
\hline \multirow{3}{*}{$\begin{array}{c}\text { Flexion } \\
\text { angle } \\
\left({ }^{\circ}\right)\end{array}$} & \multirow{2}{*}{\multicolumn{2}{|c|}{$\begin{array}{l}\text { Peak contact } \\
\text { stress }(\mathrm{MPa})\end{array}$}} & \multirow{2}{*}{\multicolumn{2}{|c|}{$\begin{array}{l}\text { Mean contact } \\
\text { stress (MPa) }\end{array}$}} & \multirow{2}{*}{\multicolumn{2}{|c|}{$\begin{array}{c}\text { Contact area } \\
\left(\mathrm{mm}^{2}\right)\end{array}$}} \\
\hline & & & & & & \\
\hline & FEM & $\begin{array}{l}\text { Nakayama } \\
\text { et al. }\end{array}$ & FEM & $\begin{array}{l}\text { Nakayama } \\
\text { et al. }\end{array}$ & FEM & $\begin{array}{l}\text { Nakayama } \\
\text { et al. }\end{array}$ \\
\hline 90 & 27.3 & $25.9 \pm 1.5$ & 13.0 & $11.1 \pm 0.2$ & 42.6 & $45.1 \pm 2.1$ \\
\hline 120 & 27.7 & $32.4 \pm 0.5$ & 12.4 & $14.8 \pm 0.5$ & 38.6 & $45.1 \pm 2.1$ \\
\hline
\end{tabular}

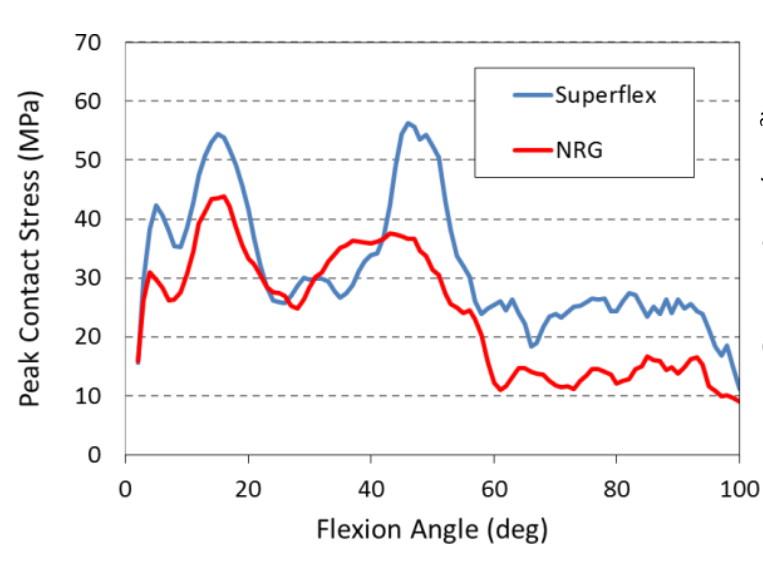

(a)

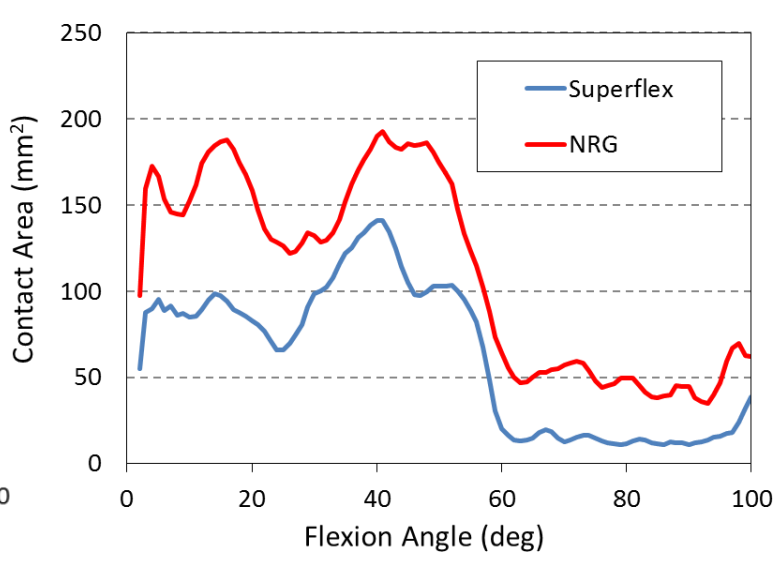

(b)

Fig. 4. Tibiofemoral contact analysis during walking gait for both models (a) Peak contact stress; and (b) Contact area.

\subsection{Stress States of Tibial Post}

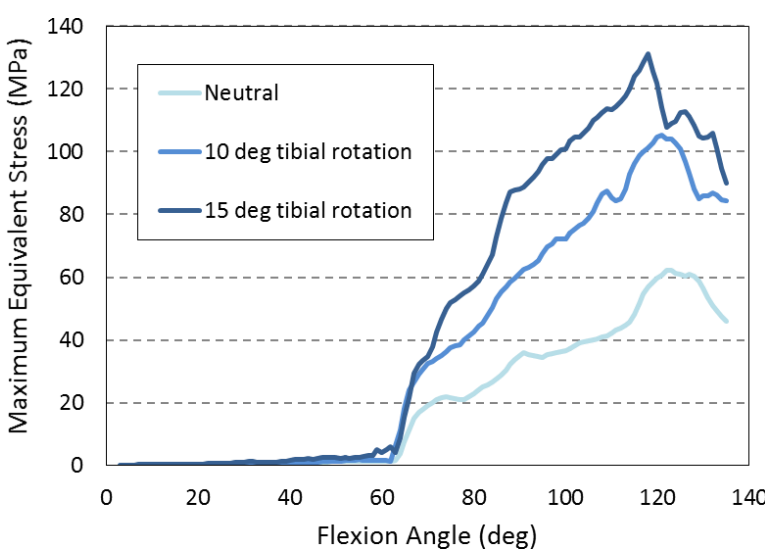

(a)

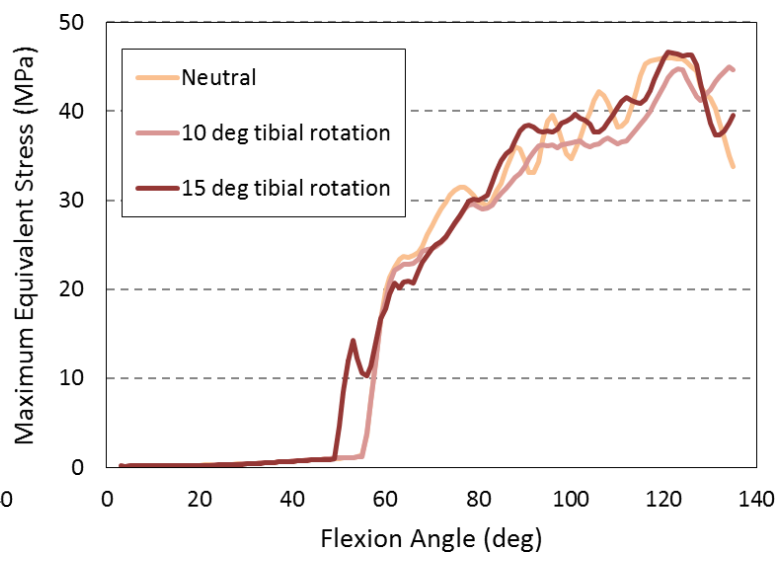

(b)

Fig. 5. Maximum equivalent stress history from 0 to $135^{\circ}$ flexion with neutral, $10^{\circ}$ and $15^{\circ}$ tibial rotation (a) Superflex; and (b) NRG.

Fig. 5 (a) and (b) shows the relationship between the maximum equivalent stress and flexion angle for 
Superflex and NRG respectively. In this study, only the maximum stress in the post of TKR was discussed as the part expose to failure during high flexion as compared to condylar part. Maximum equivalent stress increased with flexion angle for each model. It can be seen that the maximum equivalent stress increased tremendously at about $60^{\circ}$ for both TKRs shows that the post/cam contact occurs initially at this flexion angle. For Superflex, the maximum equivalent stress reached the peak values at about $120^{\circ}$ flexion with $60 \mathrm{MPa}, 110 \mathrm{MPa}$ and $130 \mathrm{MPa}$ for neutral position, $10^{\circ}$ and $15^{\circ}$ tibial rotation, respectively.

The dependence of peak values of maximum equivalent stress on tibial rotation is shown in Fig. 6 . It can be clearly noted that peak equivalent stress in post of NRG remained at about $50 \mathrm{MPa}$ with increasing tibial rotation angle. The result also shows that tibial rotation increased the peak stress in post of Superflex more than $100 \%$ between neutral position and $15^{\circ}$ tibial rotation. Distribution of equivalent stress on the tibial of both models at $120^{\circ}$ of flexion and $9^{\circ}$ of tibial angle are shown in Fig. 7.

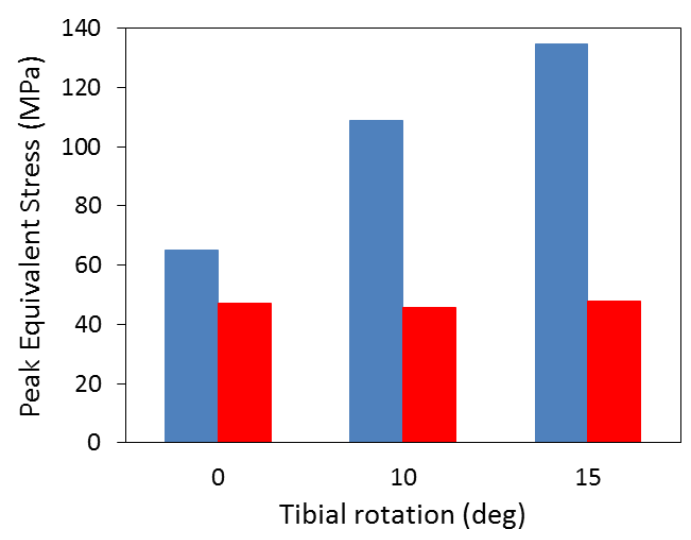

Fig. 6. Peak values of maximum equivalent stress for each type of TKA.

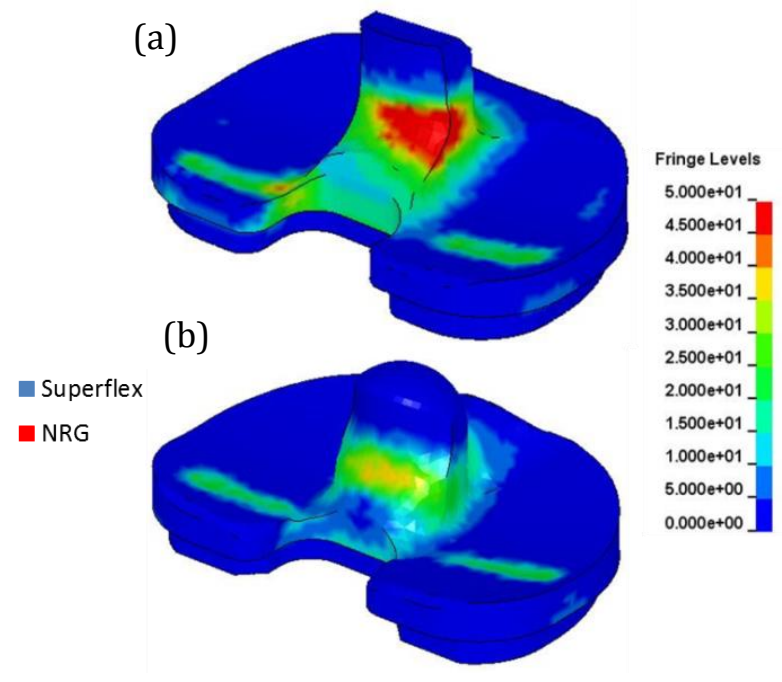

Fig. 7. Distribution of equivalent stress on the tibial insert at $120^{\circ}$ of flexion and $9^{\circ}$ of tibial angle (a) Superflex; and (b) NRG.

\section{Discussions}

Kneeling is one of the common activities among Asian especially in Japan, countries around East Asia and Middle East eg. Seiza on tatami, kneeling and squatting during prayer. Previous biomechanical investigations have shown that deep knee flexion generate significantly higher normal and anterior-posterior load in comparison to normal walking [20], [21]. It was approximated that double leg deep squatting generate up to $50.7 \%$ body weight net inferior force and $62.8 \%$ body weight net posterior load [21] while Dahlkvist et al. reported that more than 5 times bodyweight of normal load is applied to tibiofemoral joint surfaces and 2.9 to 3.5 times bodyweight of load acts tangentially during rapid descending [20]. Numerous experimental works have been carried out to study tibial rotation during deep flexion motion [17]-[19], [22]. Kinematics study on intact knee shows that the internal rotation increased with knee flexion up to $11.1^{\circ}$ [22] and $17^{\circ}$ at $137^{\circ}$ flexion for post-TKR knee [18]. Therefore, the combination of such kinetics and kinematics due to high flexion motion is predicted to be main cause of tibial post wear and damage. This hypothesis was proven by the results of the current study, showing that relatively high von Mises stress, exceeding $40 \mathrm{MPa}$ was noticeable at the tibial post for both types of TKA.

Apart from post-cam mechanics, failure of tibial post may also come from other factors such as post location and post geometry [23]. Various studies on influence of post-cam design on the mechanical 
performance of PS type TKR were reported in previous research [2], [3], [14], [24]-[26]. In general, post-cam design can be categorized into two groups, flat-on-flat and curved-on-curved [3], [14]. In this study, both TKR models are curved-on-curved types of post-cam design however, post of Superflex has larger post-to-cam radius of curvature ratio with sharper edge as compared to NRG in axial plane. Contact between post and cam at neutral tibial rotation started at $62^{\circ}$ and $56^{\circ}$ for Superflex and NRG respectively due to larger size of NRG post.

Superflex is found more sensitive towards tibial rotation than NRG due to larger edge loading during tibial rotation. The tibial rotation caused the post of tibial simultaneously sustained bending moment and torsional loading [14]. Post of Superflex has a nearly-flat posterior surface with sharp edge at both posteromedial and lateral sides. The post design has been improved by eliminating the sharp edges with rounder shape of posterior surface in NRG. The design modification reduced the edge loading, as the result, stress deviation due to tibial rotation was reduced. Fig. 8(a) and (b) show the stress distribution on tibial post in crossectional area on axial plane for both TKRs at $120^{\circ}$ flexion and $9^{\circ}$ tibial angle. It can be clearly seen that high stress concentrated around the posteromedial edge of Superflex post, in contrast, small region of stress generated on tibial post near the post-cam contact of NRG.

(a)

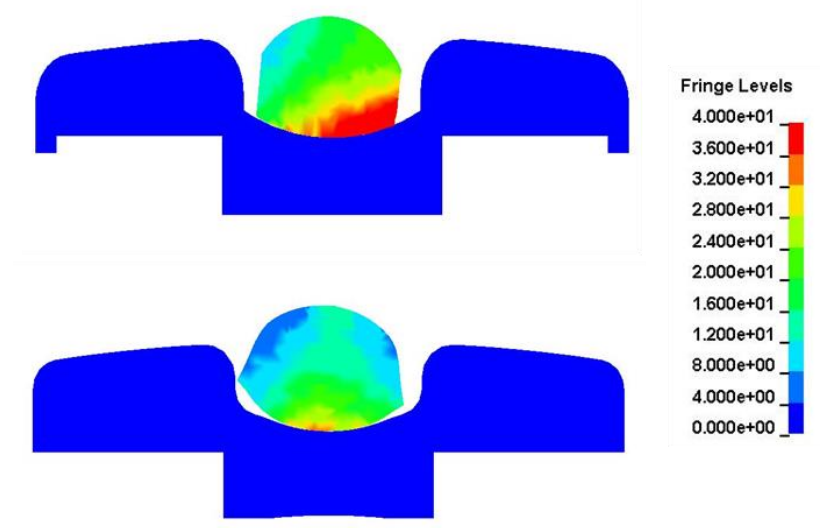

Fig. 8. Crossectional view of post-cam contact in axial plane with stress distribution at $120^{\circ}$ flexion angle and $9^{\circ}$ tibial angle for (a) Superflex; and (b) NRG.
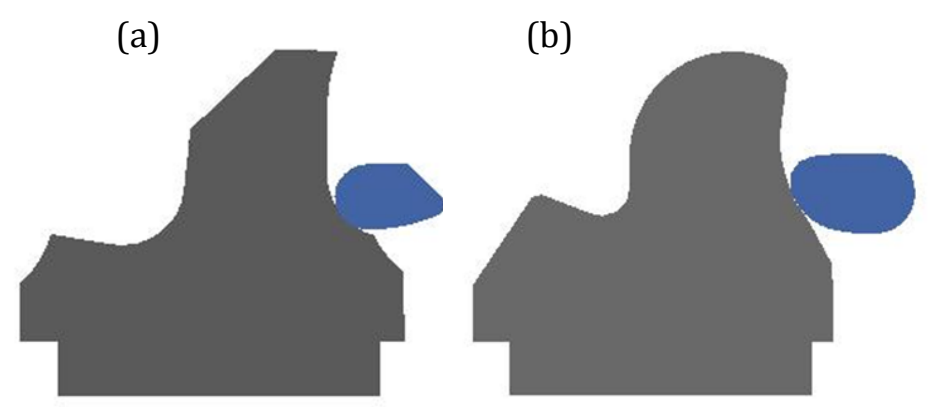

Fig. 9. Crossectional view of post-cam contact in sagittal plane at $90^{\circ}$ flexion angle with neutral rotation for (a) Superflex; and (b) NRG.

The results also show obvious variation on equivalent stress at different flexion angles. Post-cam contact of Superflex generated increasing equivalent stress from $60^{\circ}$ to $120^{\circ}$ flexion, and relatively sudden decreased from $120^{\circ}$ to $135^{\circ}$ flexion. In sagittal plane, the cam Superflex small radius of curvature at distal 
surface and increasing sharply at posterodistal surface with relatively flat posteriorly as shown in Fig. 9(a). At $120^{\circ}$ flexion, post cam contact happened at the relatively sharp curve on cam created high stress concentration on tibial post. Relatively flat posterior surface of cam reduced the equivalent stress tremendously from $120^{\circ}$ to $135^{\circ}$ of flexion angle. NRG shows almost similar trend from $60^{\circ}$ to $120^{\circ}$ flexion with lower maximum stress attained, however there was slight decrease at $135{ }^{\circ}$ of flexion. This observation was due to design of cam which oriented with large radius of curvature on posterodistal and posterior surface as in Fig. 9 (b). At neutral rotation, stress on post of Superflex was lower than NRG up to $100^{\circ}$ of flexion angle as shown in Fig. 10, however at larger flexion angle, Superflex exhibited higher stress than NRG. Post-cam contact of Superflex has good conformity at neutral position whereby the post-cam radius of curvature ratio is close to 1 in axial plane (Fig. 9(a)). The relatively flat on the distal surface of Superflex cam provide larger contact area, in contrary, relatively sharp curvature on distal surface of NRG created smaller contact area from 60 to $100^{\circ}$ flexion. Therefore, higher stress can be observed on NRG tibial post at these flexion angles.

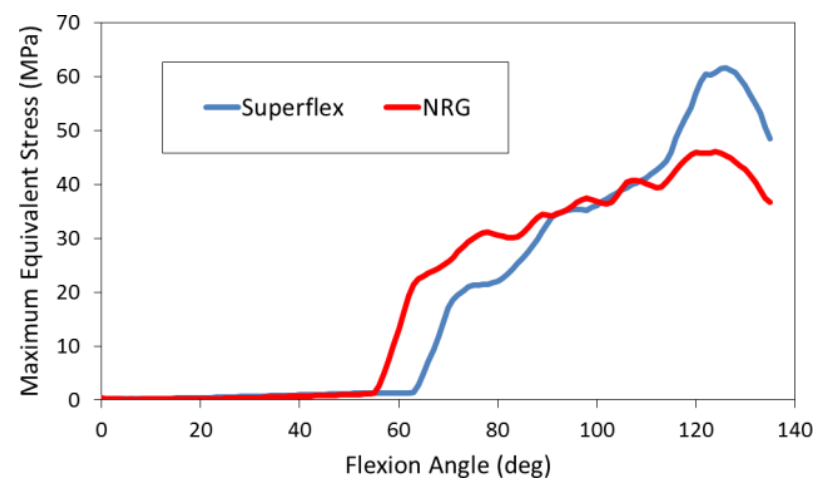

Fig. 10. Maximum equivalent stress history with neutral rotation for both models.

This study has certain limitations. The input loadings applied to the models were measured from normal patients [20] that may vary from patients with TKR. The models were subjected to flexion and tibial rotations which are linearly correlated to each other. In contrast, the higher tibial angle might occur at lower flexion angle during deep squatting. This study used a simplified model whereby two pairs of springs were utilized to represent the reaction of surrounding soft tissues [12]. The position of muscle and ligaments e.g. insertion point and relative location of ligaments on tibial and femur, were not considered in this model. Different relative orientations of ligaments insertion give different mechanical response, thus the kinetics and kinematics of knee joint will be significantly affected [27]. However, we assumed that the explicit FE model used in this study was sufficient to compare those two models of TKR.

\section{Conclusion}

In conclusion, post-cam design modification has reduced the equivalent stress generated during high flexion with tibial rotation. This study suggests that post-cam contact geometry in axial plane is important to accommodate tibial rotation and post-cam design in sagittal view is essential to accommodate deep flexional motion. The results also show that radius of curvature of tibial post relative to cam of femoral component on both axial plane and sagittal plane give significant effect on the stress state of TKR post. This study could give general guideline on the selection of TKR by surgeon as the range of motion may vary for different patient. Modification on the NRG TKA based on post-cam radius of curvature ratio in both axial and sagittal plane has eliminated sharp edge and provided larger contact area, hence reduce edge loading and high stress concentration area to accommodate lower equivalent stress during deep flexion motion and 
high tibial rotation. This modification also has made the stress in post of Scorpio NRG less sensitive to tibial rotation.

\section{References}

[1] Kurtz, S., Ong, K., Lau, E., Mowat, F., \& Halpern, M. (2007). Projections of Primary and Revision Hip and Knee Arthroplasty in the United States from 2005 to 2030. The Journal of Bone \& Joint Surgery, 89, 780-785.

[2] Todo, M., Nagamine, R., \& Yamaguchi, S. (2007). Stress Analysis of PS Type Knee Prostheses under Deep Flexion. Journal of Biomechanical Science and Engineering, 2, 237-245.

[3] Nakayama, K., Matsuda, S., Miura, H., Higaki, H., Otsuka, K., \& Iwamoto, Y. (2005). Contact stress at the post-cam mechanism in posterior-stabilised total knee arthroplasty. Journal of Bone \& Joint Surgery, British Volume, 87-B, 483-488.

[4] Godest, A. C., Beaugonin, M., Haug, E., Taylor, M., \& Gregson, P. J. (2002). Simulation of a knee joint replacement during a gait cycle using explicit finite element analysis. Journal of Biomechanics, 35, 267-275.

[5] Willing, R., \& Kim, I. Y. (2011). Design optimization of a total knee replacement for improved constraint and flexion kinematics. Journal of Biomechanics, 44, 1014-1020.

[6] Godest, A. C., Simonis de Cloke, C., Taylor, M., Gregson, P. J., Keane, A. J., Sathasivan, S., \& Walker, P. S. (2000). A computational model for the prediction of total knee replacement kinematics in the sagittal plane. Journal of Biomechanics, 33, 435-442.

[7] Halloran, J. P., Clary, C. W., Maletsky, L. P., Taylor, M., Petrella, A. J., \& Rullkoetter, P. J. (2010). Verification of predicted knee replacement kinematics during simulated gait in the Kansas knee simulator. Journal of Biomechanical Engineering, 132, 081010.

[8] Halloran, J. P., Petrella, A. J., \& Rullkoetter, P. J. (2005). Explicit finite element modeling of total knee replacement mechanics. Journal of Biomechanics, 38, 323-331.

[9] Laz, P. J., Pal, S., Halloran, J. P., Petrella, A. J., \& Rullkoetter, P. J. (2006). Probabilistic finite element prediction of knee wear simulator mechanics. Journal of Biomechanics, 39, 2303-2310.

[10] O'Brien, S., Luo, Y., Wu, C., Petrak, M., Bohm, E., \& Brandt, J. M. (2013). Computational development of a polyethylene wear model for the articular and backside surfaces in modular total knee replacements. Tribology International, 59, 284-291.

[11] Sathasivam, S., \& Walker, P. S. (1997). A computer model with surface friction for the prediction of total knee kinematics. Journal of Biomechanics, 30, 177-184.

[12] Sathasivam S., \& Walker, P. S. (1998). Computer model to predict subsurface damage in tibial inserts of total knees. Journal of Orthopaedic Research, 16, 564-571.

[13] Taylor M., \& Barrett, D. S. (2003). Explicit Finite Element Simulation of Eccentric Loading in Total Knee Replacement. Clinical Orthopaedics and Related Research, 414, 162-171.

[14] Huang, C. H., Liau, J. J., Huang, C. H., \& Cheng, C. K. (2006). Influence of Post-cam Design on Stresses on Posterior-stabilized Tibial Posts. Clinical Orthopaedics and Related Research, 450, 150-156.

[15] Innocenti, B., Pianigiani, S., Labey, L., Victor, J., \& Bellemans, J. (2011). Contact forces in several TKA designs during squatting: A numerical sensitivity analysis. Journal of Biomechanics, 44, 1573-1581.

[16] Morra, E. A., \& Greenwald, A. S. (2005). Polymer Insert Stress in Total Knee Designs During High-Flexion Activities: A Finite Element Study. The Journal of Bone \& Joint Surgery, 87, 120-124.

[17] Ueo, T., Kihara, Y., Ikeda, N., Kawai, J., Nakamura, K., \& Hirokawa, S. (2011). Deep Flexion-Oriented Bisurface-Type Knee Joint and Its Tibial Rotation That Attributes Its High Performance of Flexion. The Journal of Arthroplasty, 26, 476-482. 
[18] Kanekasu, K., Banks, S. A., Honjo, S., Nakata, O., \& Kato, H. (2004). Fluoroscopic analysis of knee arthroplasty kinematics during deep flexion kneeling. The Journal of Arthroplasty, 19, 998-1003.

[19] Nakagawa, S., Kadoya, Y., Todo, S., Kobayashi, A., Sakamoto, H., Freeman, M. A. R., \& Yamano, Y. (2000). Tibiofemoral movement 3: full flexion in the living knee studied by MRI," Journal of Bone \& Joint Surgery, British Volume, 82-B, 1199-1200.

[20] Dahlkvist, N. J., Mayo, P., \& Seedhom, B. B. (1982). Forces during Squatting and Rising from a Deep Squat. Engineering in Medicine, 11, 69-76.

[21] Nagura, T., Dyrby, C. O., Alexander, E. J., \& Andriacchi, T. P. (2002). Mechanical loads at the knee joint during deep flexion. Journal of Orthopaedic Research, 20, 881-886.

[22] Li, G., Zayontz, S., DeFrate, L. E., Most, E., Suggs, J. F., \& Rubash, H. E. (2004). Kinematics of the knee at high flexion angles: an in vitro investigation. Journal of Orthopaedic Research, 22, 90-95.

[23] Puloski, S. K. T., McCalden, R. W., MacDonald, S. J., Rorabeck, C. H., \& Bourne, R. B. (2001). Tibial Post Wear in Posterior -Stabilized Total Knee Arthroplasty An Unrecognized Source of Polyethylene Debris. The Journal of Bone \& Joint Surgery, 83, 390-390.

[24] Akasaki, Y., Matsuda, S., Shimoto, T., Miura, H., Higaki, H., \& Iwamoto, Y. (2008). Contact Stress Analysis of the Conforming Post-Cam Mechanism in Posterior-Stabilized Total Knee Arthroplasty. The Journal of Arthroplasty, 23, 736-743.

[25] Hamai, S., Miura, H., Higaki, H., Matsuda, S., Shimoto, T., Sasaki, K., Yoshizumi, M., Okazaki, K., Tsukamoto, N., \& Iwamoto, Y. (2008). Kinematic analysis of kneeling in cruciate-retaining and posterior-stabilized total knee arthroplasties. Journal of Orthopaedic Research, 26, 435-442.

[26] Huang, C. H., Liau, J. J., Huang, C. H., \& Cheng, C. K. (2007). Stress analysis of the anterior tibial post in posterior stabilized knee prostheses. Journal of Orthopaedic Research, 25, 442-449.

[27] Momersteeg, T. J. A., Blankevoort, L., Huiskes, Kooloos, R., J. G. M., Kauer, J. M. G., \& Hendriks, J. C. M. (1995). The effect of variable relative insertion orientation of human knee bone-ligament-bone complexes on the tensile stiffness. Journal of Biomechanics, 28, 745-752.

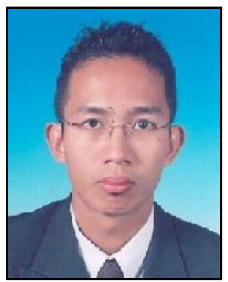

Mohd Afzan Mohd Anuar is a PhD student in biomechanics at Interdisciplinary Graduate School of Engineering Sciences, Kyushu University, Japan. He obtained his bachelor and master's degree in mechanical engineering from Universiti Kebangsaan Malaysia, Malaysia. He is currently working as a senior lecturer at Faculty of Mechanical Engineering, Universiti Teknologi MARA, Shah Alam, Selangor, Malaysia. His current research interest is biomechanics of knee arthropalsty

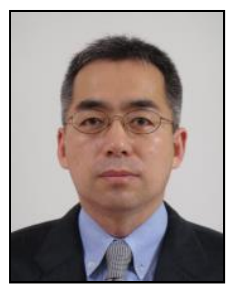

Mitsugu Todo obtained his bachelor and master's degrees of engineering from Kyushu University, Japan, and PhD from The Ohio State University, The United States of America. He is currently an associate professor of the Research Institute for Applied Mechanics, Kyushu University, and working on biomaterials for osteochondral tissue engineering and biomechanics of orthopedic implants. 